\title{
Risk factors of opioid use associated with an enhanced-recovery programme after total knee arthroplasty
}

\author{
Guorui Cao ${ }^{1 \dagger}$, Shiqi Xiang ${ }^{2 \dagger}$, Minglu Yang ${ }^{1}$, Songtao Quan ${ }^{1}$, Junna Yao ${ }^{1}$, Litao Cai ${ }^{1}$, Wei Feng ${ }^{1}$, Xiuli Yang ${ }^{3}$, \\ Hong $\mathrm{Xu}^{3}$, Zeyu Huang ${ }^{3}$, Shaoyun Zhang ${ }^{4}$, Chen Yue ${ }^{1}$, Honglue Tan ${ }^{1}$ and Fuxing Pei ${ }^{3^{*}} \mathbb{D}$
}

\begin{abstract}
Background: Characterizing the impacts of postoperative opioid use on total knee arthroplasty (TKA) patients may help optimize the pain management after TKA. The aim of the study is to examine the prevalence and risk factors for opioid use with an enhanced-recovery programme after primary TKA.

Methods: We identified 361 patients undergoing TKA, and separated those on the basis of whether to receive opioid use after surgery. Themultivariate logistic regression model was used to identify independent risk factors for opioid use after primary TKA. Length of stay (LOS) and postoperative complications were also recorded and compared.

Results: The prevalence of opioid use after primary TKA was $23.0 \%$. The significant risk factor was the longer operative time (OR [odds ratio] $=1.017,95 \% \mathrm{Cl}$ [confidence interval] $=1.001$ to $1.032, p=0.034$ ) and the protective factor was the utilization of tranexamic acid $(\mathrm{OR}=0.355,95 \% \mathrm{Cl}=0.161$ to $0.780, p=0.010)$. In addition, the $\mathrm{LOS}$ was longer in opioid group $(p<0.05)$.

Conclusion: Considering the adverse health effects of opioid use, strategies need to be developed to prevent persistent opioid use after TKA. Reducing operative time and the application of tranexamic acid could lower the risk of opioid use with an enhanced-recovery programme after primary TKA.
\end{abstract}

Keywords: Opioid, Risk factors, Total knee arthroplasty, Length of stay

\section{Introduction}

Total knee arthroplasty (TKA) is a common procedure to treat advanced osteoarthritis of the knee and the number of TKA is growing annually $[1,2]$. Due to the emergence of the significant pain in the early postoperative period following TKA,the pain management has been considered a challenging issue $[3,4]$. Appropriate pain management consist of patient education, physical therapy and

\footnotetext{
*Correspondence: peifuxingwestchina@163.com

${ }^{\dagger}$ Guorui Cao and Shiqi Xiang contributed equally to this manuscript.

${ }^{3}$ Department of Orthopaedic surgery, West China Hospital, Sichuan

University, 37\# Guoxue Road, Chengdu, Sichuan Province 610041,

People's Republic of China

Full list of author information is available at the end of the article
}

medications, including acetaminophen, non-steroidal anti-inflammatory drugs (NSAIDs) and opioid [5].

Opioid has been proposed as one of the effective methods to treat choric pain after TKA, many patients in America even receive opioid for more than 3 months to control pain postoperatively [6]. However, it is reported that postoperative opioid utilization could also cause a series of side effects, such as delayed rehabilitation, higher revision risk, opioid dependency and withdrawal [7-9]. , Despite it, as the strongest analgesic agent for treating the postsurgical patients, opioid still be considered as the important remedial pain management drug for TK A[4]..

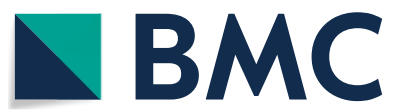

(c) The Author(s) 2021. Open Access This article is licensed under a Creative Commons Attribution 4.0 International License, which permits use, sharing, adaptation, distribution and reproduction in any medium or format, as long as you give appropriate credit to the original author(s) and the source, provide a link to the Creative Commons licence, and indicate if changes were made. The images or other third party material in this article are included in the article's Creative Commons licence, unless indicated otherwise in a credit line to the material. If material is not included in the article's Creative Commons licence and your intended use is not permitted by statutory regulation or exceeds the permitted use, you will need to obtain permission directly from the copyright holder. To view a copy of this licence, visit http://creativecommons.org/licenses/by/4.0/. The Creative Commons Public Domain Dedication waiver (http://creativeco mmons.org/publicdomain/zero/1.0/) applies to the data made available in this article, unless otherwise stated in a credit line to the data. 
Previous researchers used opioid as a routine means of analgesia and the postoperative utilization rate was approximately $90 \%$. The risk factors for prolonged opioid use and opioid-related adverse drug events after TKA were extensively studied in previous studies [10-13]. With the development of enhanced recovery following joint surgery, preemptive analgesia, multimodal analgesia as well as the application of tranexamic acid (TXA) and dexamethasone relieved pain intensity after TKA. Considering the side effects of opioid, opioid has been only taken as a remedy when postoperative pain control was poor in our clinic center. Since there is no perfect pain management protocol, it is quite necessary to investigate the relationship between TKA and the usage of opioid. The prevalence and related factors associated with whether or not use opioid after primary TKA are still poorly studied. Therefore, we perform this retrospective cohort study to investigate the risk factors associated with postoperative opioid use following primary TKA.

\section{Materials and methods Study design and study sample}

We conducted a single-center retrospective cohort study and enrolled patients undergoing primary TKA owing to osteoarthritis and rheumatoid arthritis from June 2016 to January 2019. The study was approved by the hospital's Institutional Review Board. This study was reported using the Strengthening the Reporting of Observational Studies in Epidemiology guidelines [14].

The patients were excluded with any of the following situations: cardiovascular problems (myocardial infarction, cardiac insufficiency, and other serious heart diseases evaluated by physicians), history of chronic musculoskeletal pain or other types of chronic pain, history of cancer, and incomplete medical records. Overall, 361 patients were included in this study. The enrolled patients were divided into the opioid group or non-opioid group according to whether patients received opioid drugs to control pain after primary TKA.

\section{Surgical Technique and Perioperative protocol}

All the surgeries were performed under general anesthesia by the same senior surgeon, through a midline skin incision, medial parapatellar approach, and a measured resection technique. The prosthesis was cemented posterior-stabilized prosthetic design with patellar resurfacing. $200 \mathrm{mg}$ ropivacaine diluted in $100 \mathrm{~mL}$ of normal saline solution was injected periarticularly when the deep fascia was sutured. No tourniquet, nerve block, intravenous patient-controlled analgesia or blood salvage system were used. The operative time and intraoperative blood loss were recorded carefully.

All patients received enhanced recovery protocols, including blood management, sleep management and so on $[15,16]$. The detailed strategy was showed in Table 1. The physical prophylaxis and chemoprophylaxis were applied to prevent DVT and PE based on our previous study [3]. The TXA $(20 \mathrm{mg} / \mathrm{kg})$ was administered intravenously 5-10 minutes before skin incision, along with 1 g intravenous TXA (diluted in $100 \mathrm{~mL}$ of normal saline solution) at 3, 6, 12, and 24 hours postoperatively.

Routinely, all patients received oral Celecoxib (200 mg twice a day, Celebrex; Pfizer, NewYork) preoperatively and oral enteric-coated diclofenac sodium (50 mg twice a day, Voltaren; Novartis, Basel, Switzerland) postoperatively for 2-4 weeks after surgery. $200 \mathrm{mg}$ ropivacaine dissolved in $60 \mathrm{~mL}$ normal saline was infiltrated around articular cavity when the prosthesis was placed. Cold pack and intermittent foot slope pump systems were given to all patients for 12-24 hours postoperatively. The Visual Analogue Scale (VAS) score was measured and recorded preoperatively and postoperative every day (Twice a day, 8 a.m. and 6 p.m.) in motion and at rest by surgeon and nurses before discharging hospital. Using opioid drug (OxyContin or oxycodone hydrochloride

Table 1 The strategy of enhanced recovery after primary TKA

\begin{tabular}{ll}
\hline & Detailed measures \\
\hline Blood Management & Correcting perioperative anemia, including iron supplement, erythropoietin and transfusion. \\
& Multiple TXA application: 5-10 min preoperatively and 3, 6, 12, and 24 hour postoperatively. \\
Pain Management & Preoperative education \\
& Preemptive analgesia: oral Celecoxib preoperatively more than 1 week. \\
& Multimodal analgesia: oral NSAIDs postoperatively 2-4 weeks and 200 mg ropivacaine local \\
infiltration around articular cavity intraoperatively. & Remedy: Opioid. \\
& Preoperative early-stage feeding:receiving carbohydrate powder 2 hour and protein powder \\
& 6 hour dissolved in normal saline before the induction of anesthesia. \\
& Postoperative diet starting 2-4 hours after surgery.
\end{tabular}


sustained release tablets) once or more after surgery was defined as opioid use. The opioid was applied when the VAS score was $\geq 5$ in motion or $\geq 3$ in rest. The dosage was $10 \mathrm{mg}$ twice a day, increasing gradually when pain control was poor (VAS score was $\geq 5$ in motion or $\geq 3$ in rest) and the maximum of dosage was $100 \mathrm{mg}$. The surgeon decided when to prescribe opioids according to pain control situation.

\section{Outcome Measurements}

The number of patients who received opioid prescriptions after TKA was the outcome of interest. Perioperative variables considered as potential risk factors, including age, gender, diagnosis, body mass index, medical comorbidities and laboratory markers were collected. In addition, the American Society of Anesthesiologists physical status classification, the application of TXA and drainage, operative time, intraoperative blood loss, length of stay (LOS) after surgery, and postoperative complications were carefully recorded.

\section{Statistical analyses}

Descriptive and univariate analyses were conducted to compare the characteristics in patients with or without opioid use after primary TKA. A multivariable logistic regression model was used to examine independent risk factor for postoperative opioid use in TKA. Factors associated with postoperative opioid use with $p<0.10$ were selected for inclusion in the multivariable model. Collinearity was checked using tolerance values $(\mathrm{p}>0.10)$. All analyses were performed using SPSS version 22.0(SPSS Inc. USA). A $p$ value of $<0.05$ was considered statistically significant.

\section{Results}

Totally, 361 patients were enrolled in the study. Among them, 83 patients received opioid drugs to control pain after primary TKA. The prevalence of opioid use was $23.0 \%$. The results of comparison between the opioid and non-opioid group were shown in Table 2. The variables that were selected for the logistic regression model after univariate analyses were smoking, TXA use, more operative time and intraoperative blood loss $(p<0.10)$.

Risk factors independently associated with opioid use was the longer operative time (OR [odds ratio] $=1.017$, $95 \% \mathrm{CI}$ [confidence interval]) $=1.001$ to $1.032, p=$ 0.034). Moreover, the administration of TXA was a protective factor for opioid use after TKA (OR $=0.355,95 \%$ $\mathrm{CI}=0.161$ to $0.780, p=0.010$ ) (Table 3).

The LOS and related complications were summarized in Table 4. The LOS in opioid group was longer than that in non-opioid group $(3.8 \pm 1.2$ vs $3.5 \pm 0.9, p=0.36)$. In addition, the incidence of nausea and vomiting was higher in opioid group ( $25.3 \%$ vs $11.2 \%, p=0.001)$. The incidence of other complications, including deep venous thrombosis, pulmonary embolism, wound complications and so on, did not reach statistical difference between the two groups $(p>0.05)$

\section{Discussion}

Postoperative persistent pain following TKA is a collective concern for surgeons and patients [17]. Effective pain management after TKA could decrease complication risk, promote earlier rehabilitation, improve patient's satisfaction and quality of life [18-20]. When conventional measures, such as NSAIDs and physical therapy, had poor efficacy, opioid drugs could be an available remedy in this study. Thus, the risk factor associated with opioid use could be responsible for the poor pain control after primary TKA. Because of the adverse effects of severe pain and opioid use, effective pain control and recognition of the risk factors for opioid use could guide provider and hospital system approach to managing postoperative pain after TKA [20, 21].

Substantial previous studies focused on the prevalence and prolonged opioid use instead of simple opioid use after TKA [10,12,13]. A retrospective cohort study performed by Robert et al indicated that younger age, preoperative NSAIDs and opioid use as well as some comorbidities were significant risk factors associated with prolonged postoperative opioid use after TKA [13]. In addition, Bedard and his colleagues determined that preoperative opioid use, a higher comorbidity score, rheumatoid arthritis, smoking and the benzodiazepine utilization were strong predictors for persistent opioid use postoperatively following TKA [10]. The research conducted by Kim et al reached a similar conclusion [12]. To our best knowledge, this study is the first one to determine the risk factors for the postoperative opioid use following primary TKA. Following an enhanced-recovery programme, the longer operative time is a risk factor while TXA use is a protective risk for opioid use after primary TKA.

It was reported that operative time, intraoperative blood loss and postoperative pain in TKA were in close positive correlation. Extended operative time could lead to more intraoperative blood loss, further resulting in increasing pain after TKA [22, 23]. Consequently, reducing operative time and blood loss was important step for postoperative pain care. Besides, smoking might also increase the risk for opioid use after TKA $(p=0.054)$. It was noted that smoking could cause more perioperative blood loss and higher complication risks [24]. Cryar et al demonstrated that preoperative smoking was the risk factor for narcotic use after TKA [25]. In addition, smoking 
Table 2 The comparison of patients with or without opioid use following primary TKA

\begin{tabular}{|c|c|c|c|}
\hline Baseline Characteristic & Opioid group, $\mathrm{N}=83$ & Non-opioid group, $\mathrm{N}=\mathbf{2 7 8}$ & $p$ \\
\hline \multicolumn{4}{|l|}{ Demographic characteristics } \\
\hline $\operatorname{Age}(\bar{X} \pm S)$ & $66.1 \pm 8.9$ & $66.0 \pm 7.7$ & 0.897 \\
\hline Sex (male/female) & $19 / 64$ & $45 / 233$ & 0.160 \\
\hline $\operatorname{BMI}\left(\bar{X} \pm \mathrm{S}, \mathrm{kg} / \mathrm{m}^{2}\right)$ & $25.6 \pm 3.5$ & $25.4 \pm 3.6$ & 0.880 \\
\hline Diagnosis (RA/OA) & $5 / 78$ & $8 / 270$ & 0.177 \\
\hline Preoperative HSS & $48.7 \pm 8.9$ & $49.3 \pm 8.2$ & 0.582 \\
\hline Preoperative VAS & $4.9 \pm 0.7$ & $4.9 \pm 0.8$ & 0.578 \\
\hline Preoperative varus (N, \%) & $45(54.2 \%)$ & $149(53.6 \%)$ & 0.921 \\
\hline Preoperative valgus (N, \%) & $8(9.6 \%)$ & $30(10.8 \%)$ & 0.764 \\
\hline \multicolumn{4}{|l|}{ Comorbidities (N, \%) } \\
\hline Smoking & $10(12.0 \%)$ & $17(6.1 \%)$ & 0.071 \\
\hline Alcohol & $5(6.0 \%)$ & $16(5.8 \%)$ & 0.927 \\
\hline Hypertension & $48(57.8 \%)$ & $167(73.3 \%)$ & 0.715 \\
\hline Diabetes & $10(12.0 \%)$ & $48(21.1 \%)$ & 0.256 \\
\hline Coronary heart disease & $10(12.0 \%)$ & $20(7.2 \%)$ & 0.160 \\
\hline Preoperative anemia & 19 (22.9\%) & $62(22.3 \%)$ & 0.910 \\
\hline COPD & $8(9.6 \%)$ & $22(7.9 \%)$ & 0.617 \\
\hline \multicolumn{4}{|l|}{ Preoperative laboratories } \\
\hline $\mathrm{HB}(\mathrm{g} / \mathrm{L})$ & $131.3 \pm 13.4$ & $130.7 \pm 12.9$ & 0.698 \\
\hline Hct & $39.7 \pm 3.7$ & $39.9 \pm 3.6$ & 0.746 \\
\hline Albumin (g/L) & $42.8 \pm 3.2$ & $43.4 \pm 3.2$ & 0.191 \\
\hline $\operatorname{ESR}(\mathrm{mm} / \mathrm{h})$ & $24.0 \pm 16.3$ & $27.0 \pm 18.0$ & 0.259 \\
\hline CRP (mg/L) & $3.5 \pm 4.28$ & $3.6 \pm 3.0$ & 0.748 \\
\hline IL-6 (mg/L) & $4.3 \pm 2.9$ & $4.2 \pm 4.1$ & 0.771 \\
\hline Platelet $\left(* 10^{9} / \mathrm{L}\right)$ & $183.5 \pm 68.6$ & $187.6 \pm 59.6$ & 0.635 \\
\hline PT (sec) & $11.4 \pm 0.7$ & $11.3 \pm 1.0$ & 0.670 \\
\hline APTT (sec) & $27.7 \pm 3.1$ & $27.5 \pm 3.3$ & 0.751 \\
\hline \multicolumn{4}{|l|}{ Operative variables } \\
\hline ASA class (N, \%) & & & 0.789 \\
\hline $1-2$ & $70(84.3 \%)$ & $231(83.1 \%)$ & \\
\hline$\geq 3$ & $13(15.7 \%)$ & $47(16.9 \%)$ & \\
\hline Drainage use $(\mathrm{N}, \%)$ & $22(26.5 \%)$ & $88(31.7 \%)$ & 0.371 \\
\hline TXA use (N, \%) & 71 (85.5\%) & $259(93.2 \%)$ & $0.030^{*}$ \\
\hline Dexamethasone use (N,\%) & $65(78.3 \%)$ & $234(84.2 \%)$ & 0.214 \\
\hline Operative time (min) & $80.4 \pm 16.2$ & $76.7 \pm 15.0$ & 0.056 \\
\hline Intraoperative blood loss (mL) & $127.4 \pm 33.0$ & $104.6 \pm 29.2$ & $<0.001^{*}$ \\
\hline
\end{tabular}

BMI: body mass index; RA: rheumatoid arthritis; OA: osteoarthritis; HSS: hospital for special surgery knee score; VAS: visual analogue scale; COPD: chronic obstructive pulmonary disease; HB: hemoglobin; Hct: hematocrit; ESR: erythrocyte sedimentation rate; CRP: C reaction protein; IL-6: interleukin-6; PT: prothrombin time; APTT: activated partial thrombin time; ASA, American Society of Anesthesiologists; TXA: Tranexamic acid.

$p$ value calculated using independent t-test, Pearson chi-square test or Fisher exact test.

*Significant difference

was also a strong risk factor for persistent opioid use after primary TKA [12].

In this study, we found that application of TXA could reduce the risk of persistent pain and the risk of opioid use after TKA. As an antifibrinolytic agents, TXA could inhibit hyperfibrinolysis, reduce blood loss, alleviate joint swelling and relieve pain [26, 27]. Related studies also showed that patients could have less pain, less knee swelling and better knee function with the usage of multiple boluses of TXA following TKA [27, 28]. In addition to reducing blood loss, TXA have a synergistic effect to relieve pain after orthopaedic procedures. Currently, the application of TXA is an essential measure for blood management in enhanced recovery protocols. 
Table 3 Logistic regression models for opioid use following primary TKA

\begin{tabular}{llll}
\hline Parameter & Odds Ratio & $\begin{array}{l}\text { 95\% Confidence } \\
\text { Interval }\end{array}$ & $\boldsymbol{p}$ \\
\hline Smoking & 2.274 & $0.988-5.234$ & 0.054 \\
TXA use & 0.355 & $0.161-0.780$ & $0.010^{*}$ \\
Operative time & 1.017 & $1.001-1.032$ & $0.034^{*}$ \\
\hline
\end{tabular}

TXA: Tranexamic acid.

*Significant difference

Table 4 LOS, expenses and Complications

\begin{tabular}{llll}
\hline Variables & $\begin{array}{l}\text { Opioids } \\
\text { group, } \mathbf{N}=\mathbf{8 3}\end{array}$ & $\begin{array}{l}\text { Non-opioids } \\
\text { group, } \mathbf{N}=\mathbf{2 7 8}\end{array}$ & $\boldsymbol{p}$ \\
\hline LOS & $3.8 \pm 1.2$ & $3.5 \pm 0.9$ & $0.036^{*}$ \\
Transfusion & $1(1.2 \%)$ & $1(0.4 \%)$ & 1.000 \\
Death & 0 & 0 & - \\
DVT & 0 & $4(1.4 \%)$ & 0.489 \\
PE & 0 & 0 & - \\
Nausea and vomiting & $21(25.3 \%)$ & $31(11.2 \%)$ & $0.001^{*}$ \\
Cardiac infarction & $1(1.2 \%)$ & 0 & 0.230 \\
Stroke & 0 & 0 & - \\
Acute renal failure & 0 & 0 & - \\
Wound complications & $16(19.3 \%)$ & $37(13.3 \%)$ & 0.178 \\
Readmission & $2(2.4 \%)$ & $2(0.7 \%)$ & 0.228 \\
\hline
\end{tabular}

LOS: length of stay; DVT: deep venous thrombosis; PE: pulmonary embolism. Wound complications included exudation, bleeding, swelling, infection and impaired wound healing.

$p$ value calculated using independent t-test, Pearson chi-square test or Fisher exact test.

The patients in opioid group sustained worse pain, longer LOS, higher incidence of nausea and vomiting after primary TKA. Opioid use was associated with increased LOS after TKA, which is consistent with previous studies $[8,12]$. Halawi et al showed that opioid-based analgesia was significantly associated with increased LOS [8]. Another literature supported this result, showing that opioid use could lead to longer hospitalizations [11]. In addition, nausea and vomiting, which could increase complication risk and delay postoperative recovery, may be the side effects of opioid use [29]. So, it is necessary to take measures to prevent nausea and vomiting.

There are some limitations in this study. Firstly, we do not enroll preoperative medication history, such as sedative and opioid because related case data is incomplete. It was reported that preoperative benzodiazepine and opioid use was strong predictor for postoperative opioid use in TKA [10, 12]. Secondly, as a retrospective study, all data is from electronic medical record system and the level of evidence is still low. In addition, the follow-up time is only 30 days while chronic pain after TKA may continue more than 6 months [12]. So, the follow-up time in this study is insufficient to evaluate entire patients with opioid use after primary TKA. In a word, further studies with higher evidence level are requisite.

\section{Conclusion}

Considering the adverse health effects of opioid use, strategies need to be developed to prevent persistent opioid use. Reducing operative time and the application of tranexamic acid could lower the risk of opioid use with an enhanced-recovery programme after primary TKA.

\section{Abbreviations}

TKA: Total knee arthroplasty; LOS: Length of stay; OR: Odds ratio; Cl: confidence interval; NSAIDs: Non-steroidal anti-inflammatory drugs; DVT: Deep venous thrombosis; PE: Pulmonary embolism; TXA: Tranexamic acid; VAS: Visual Analogue Scale.

\section{Acknowledgements}

This work was supported by Department of Knee Injury, Luoyang Orthopedic Hospital of Henan Province and Department of Orthopaedic surgery, West China Hospital, Sichuan University. We thank Dr. Shiqi Xiang, from Department of Orthopedics, the Second Xiangya Hospital of Central South University for the re-interpretation of data and finalizing of the revision.

\section{Authors' contributions}

GC collected, processed, analyzed and interpreted data and wrote the manuscript. SX analyzed and interpreted data, edited the manuscript finalized the revise. MY collected data and edited the manuscript. SQ collected data and reviewed the manuscript. JY analyzed and interpreted data, and edited the manuscript. LC conceptualized, interpreted data, and edited the manuscript. $W F, X Y, H X, Z H, S Z, C Y$ and $H T$ reviewed and edited the manuscript. FP conceptualized the study, interpreted data, contributed to manuscript writing and editing, and obtained funding. All authors approved the final manuscript.

\section{Funding \\ None.}

\section{Availability of data and materials}

The datasets used and/or analyzed during the current study are available from the corresponding author on reasonable request.

\section{Declarations}

\section{Ethics approval and consent to participate}

The study was a retrospective cohort study. This study was approved by the local institutional review board of West China Hospital, Sichuan University (2012-268). Written informed consent (including patients' details, images or videos) was obtained from all participants.

\section{Consent for publication}

Not applicable.

\section{Competing interests}

The authors declare that they have no competing interests.

\section{Author details}

${ }^{1}$ Department of Knee Injury, Luoyang Orthopedic Hospital of Henan Province, Orthopedic Hospital of Henan Province, Luoyang, Henan Province, People's Republic of China. ${ }^{2}$ Department of Orthopedics, The Second Xiangya Hospital of Central South University, Changsha, Hunan Province, People's Republic of China. ${ }^{3}$ Department of Orthopaedic surgery, West China Hospital, Sichuan University, 37\# Guoxue Road, Chengdu, Sichuan Province 610041, People's Republic of China. ${ }^{4}$ Department of Orthopedics, The Third Hospital 
of Mianyang, Sichuan Mental Health Center, Mianyang, Sichuan Province, People's Republic of China.

Received: 4 January 2021 Accepted: 3 December 2021

Published online: 20 December 2021

\section{References}

1. Kirksey M, Chiu YL, Ma Y, Della Valle AG, Poultsides L, Gerner P, et al. Trends in in-hospital major morbidity and mortality after total joint arthroplasty: United States 1998-2008. Anesthesia and analgesia. 2012;115(2):321-7.

2. Steele T, Eidem L, Bond J: Impact of Adoption of Smart Pump System With Continuous Capnography Monitoring on Opioid-Related Adverse Event Rates: Experience From a Tertiary Care Hospital. Journal of patient safety 2019

3. Lei YT, Xu B, Xie XW, Xie JW, Huang Q, Pei FX. The efficacy and safety of two low-dose peri-operative dexamethasone on pain and recovery following total hip arthroplasty: a randomized controlled trial. International orthopaedics. 2018:42(3):499-505.

4. Xie X, Pei F, Huang Z, Tan Z, Yang Z, Kang P. Does patellar denervation reduce post-operative anterior knee pain after total knee arthroplasty? Knee surgery, sports traumatology, arthroscopy : official journal of the ESSKA. 2015;23(6):1808-15.

5. Hochberg MC, Altman RD, April KT, Benkhalti M, Guyatt G, McGowan J, et al. American College of Rheumatology 2012 recommendations for the use of nonpharmacologic and pharmacologic therapies in osteoarthritis of the hand, hip, and knee. Arthritis care \& research. 2012;64(4):465-74.

6. Cancienne JM, Patel KJ, Browne JA, Werner BC. Narcotic Use and Total Knee Arthroplasty. The Journal of arthroplasty. 2018;33(1):113-8.

7. da Costa BR, Nüesch E, Kasteler R, Husni E, Welch V, Rutjes AW, Jüni P: Oral or transdermal opioids for osteoarthritis of the knee or hip. The Cochrane database of systematic reviews 2014(9):Cd003115.

8. Halawi MJ, Vovos TJ, Green CL, Wellman SS, Attarian DE, Bolognesi MP. Opioid-Based Analgesia: Impact on Total Joint Arthroplasty. The Journal of arthroplasty. 2015;30(12):2360-3.

9. Inacio MC, Pratt NL, Roughead EE, Paxton EW, Graves SE. Opioid use after total hip arthroplasty surgery is associated with revision surgery. BMC musculoskeletal disorders. 2016;17:122.

10. Bedard NA, Pugely AJ, Westermann RW, Duchman KR, Glass NA, Callaghan JJ. Opioid Use After Total Knee Arthroplasty: Trends and Risk Factors for Prolonged Use. The Journal of arthroplasty. 2017;32(8):2390-4.

11. Jones MR, Kramer ME, Beutler SS, Kaye AD, Rao N, Brovman EY, et al. The Association Between Potential Opioid-Related Adverse Drug Events and Outcomes in Total Knee Arthroplasty: A Retrospective Study. Advances in therapy. 2020;37(1):200-12.

12. Kim SC, Choudhry N, Franklin JM, Bykov K, Eikermann M, Lii J, et al. Patterns and predictors of persistent opioid use following hip or knee arthroplasty. Osteoarthritis and cartilage. 2017;25(9):1399-406.

13. Namba RS, Singh A, Paxton EW, Inacio MCS. Patient Factors Associated With Prolonged Postoperative Opioid Use After Total Knee Arthroplasty. The Journal of arthroplasty. 2018;33(8):2449-54.

14. von Elm E, Altman DG, Egger M, Pocock SJ, Gøtzsche PC, Vandenbroucke JP. The Strengthening the Reporting of Observational Studies in Epidemiology (STROBE) statement: guidelines for reporting observational studies. Lancet (London, England). 2007;370(9596):1453-7.

15. Ripolles-Melchor J, Abad-Motos A, Diez-Remesal Y, Aseguinolaza-Pagola M, Padin-Barreiro L, Sanchez-Martin R, Logrono-Egea M, Catala-Bauset JC, Garcia-Orallo S, Bisbe E et al: Association Between Use of Enhanced Recovery After Surgery Protocol and Postoperative Complications in Total Hip and Knee Arthroplasty in the Postoperative Outcomes Within Enhanced Recovery After Surgery Protocol in Elective Total Hip and Knee Arthroplasty Study (POWER2). JAMA surgery 2020:e196024.

16. Shen $B$, Weng $X$, Liao $R$, et al. Enhanced recovery of hip and knee arthroplasty in China-a consensus of experts on perioperative pain and sleep management. Chinese Journal of bone and joint surgery. 2016;9(02):91-7.

17. Nilsdotter AK, Toksvig-Larsen S, Roos EM. Knee arthroplasty: are patients' expectations fulfilled? A prospective study of pain and function in 102 patients with 5-year follow-up. Acta orthopaedica. 2009;80(1):55-61.
18. Elmallah RK, Chughtai M, Khlopas A, Newman JM, Stearns KL, Roche M, et al. Pain Control in Total Knee Arthroplasty. The journal of knee surgery. 2018:31(6):504-13.

19. Gonzales J, Lovald ST, Lau EC, Ong KL. Risk of Opioid-Related Adverse Events After Primary and Revision Total Knee Arthroplasty. Journal of surgical orthopaedic advances. 2018;27(2):148-54.

20. Shafi S, Collinsworth AW, Copeland LA, Ogola GO, Qiu T, Kouznetsova M, et al. Association of Opioid-Related Adverse Drug Events With Clinical and Cost Outcomes Among Surgical Patients in a Large Integrated Health Care Delivery System. JAMA surgery. 2018;153(8):757-63.

21. Baratta JL, Gandhi K, Viscusi ER. Perioperative pain management for total knee arthroplasty. Journal of surgical orthopaedic advances. 2014;23(1):22-36

22. Friedman RJ. Limit the bleeding, limit the pain in total hip and knee arthroplasty. Orthopedics. 2010;33(9 Suppl):11-3.

23. Hrnack SA, Skeen N, Xu T, Rosenstein AD. Correlation of body mass index and blood loss during total knee and total hip arthroplasty. American journal of orthopedics (Belle Mead, NJ). 2012;41(10):467-71.

24. Manchikanti L, Singh A. Therapeutic opioids: a ten-year perspective on the complexities and complications of the escalating use, abuse, and nonmedical use of opioids. Pain physician. 2008;11 (2 Suppl):S63-88.

25. Cryar KA, Hereford T, Edwards PK, Siegel E, Barnes CL, Mears SC. Preoperative Smoking and Narcotic, Benzodiazepine, and Tramadol Use are Risk Factors for Narcotic Use After Hip and Knee Arthroplasty. The Journal of arthroplasty. 2018;33(9):2774-9.

26. Hogan CA, Golightly LK, Phong S, Dayton MR, Lyda C, Barber GR. Perioperative blood loss in total hip and knee arthroplasty: Outcomes associated with intravenous tranexamic acid use in an academic medical center. SAGE open medicine. 2016;4:2050312116637024.

27. Xie J, Ma J, Yao H, Yue C, Pei F. Multiple Boluses of Intravenous Tranexamic Acid to Reduce Hidden Blood Loss After Primary Total Knee Arthroplasty Without Tourniquet: A Randomized Clinical Trial. The Journal of arthroplasty. 2016;31(11):2458-64.

28. Lei Y, Huang Q, Huang Z, Xie J, Chen G, Pei F. Multiple-Dose Intravenous Tranexamic Acid Further Reduces Hidden Blood Loss After Total Hip Arthroplasty: A Randomized Controlled Trial. The Journal of arthroplasty. 2018;33(9):2940-5.

29. Ryu JH, Jeon YT, Min B, Hwang JY, Sohn HM. Effects of palonosetron for prophylaxis of postoperative nausea and vomiting in high-risk patients undergoing total knee arthroplasty: A prospective, randomized, doubleblind, placebo-controlled study. PloS one. 2018;13(5):e0196388.

\section{Publisher's Note}

Springer Nature remains neutral with regard to jurisdictional claims in published maps and institutional affiliations.

\footnotetext{
Ready to submit your research? Choose BMC and benefit from:

- fast, convenient online submission

- thorough peer review by experienced researchers in your field

- rapid publication on acceptance

- support for research data, including large and complex data types

- gold Open Access which fosters wider collaboration and increased citations

- maximum visibility for your research: over 100M website views per year
}

At BMC, research is always in progress.

Learn more biomedcentral.com/submissions 